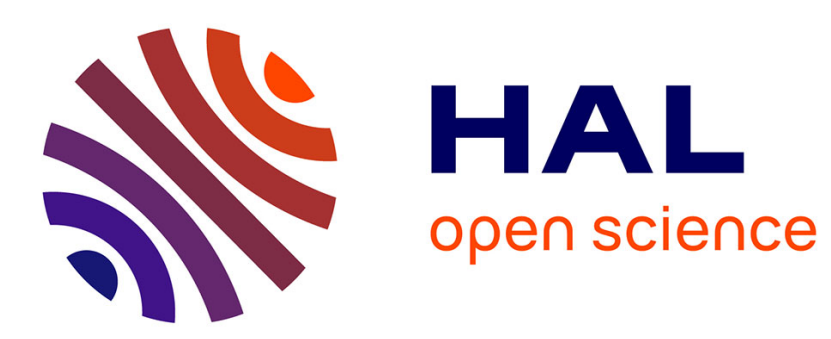

\title{
La mort de l'animal dans l'imaginaire catholique (France, XVIIe-XXe siècles)
}

\author{
Eric Baratay
}

\section{To cite this version:}

Eric Baratay. La mort de l'animal dans l'imaginaire catholique (France, XVIIe-XXe siècles). Revue de l'histoire des religions, 1995, 212 (4), pp.453-476. 10.3406/rhr.1995.1251 . halshs-01569469

\section{HAL Id: halshs-01569469 \\ https://shs.hal.science/halshs-01569469}

Submitted on 26 Jul 2017

HAL is a multi-disciplinary open access archive for the deposit and dissemination of scientific research documents, whether they are published or not. The documents may come from teaching and research institutions in France or abroad, or from public or private research centers.
L'archive ouverte pluridisciplinaire HAL, est destinée au dépôt et à la diffusion de documents scientifiques de niveau recherche, publiés ou non, émanant des établissements d'enseignement et de recherche français ou étrangers, des laboratoires publics ou privés. 


\section{Éric Baratay}

\section{La mort de l'animal dans l'imaginaire catholique (France, XVIle- XXe siècle) \\ In: Revue de l'histoire des religions, tome 212 n4, 1995. pp. 453-476.}

\section{Résumé}

Pour la majorité des catholiques, et notamment du clergé, la mort de l'animal n'est qu'un phénomène banal. Son absence évidente de survie permet de le tuer sans appréhension pour s'en servir abondamment. Les recommandations sporadiques sur la modération de la violence ne modifient pas ce schéma général. Ce modèle est de plus en plus contesté par un courant minoritaire qui émerge au XIXe siècle, même si des témoignages montrent qu'il existait auparavant à l'état latent. Composé essentiellement de laïcs, il considère que la mort de l'animal est le plus souvent injuste et qu'une forme de survie doit la compenser.

\section{Abstract}

The death of an animal in Catholic Imagination (France, 17th - 20th centuries)

For most Catholics, in particular the clergy, the death of an animal is no more than a commonplace event. Since it obviously has no life after death it can be killed without apprehension and used in abundance. The occasional recommendation to use less violent methods of killing did not alter this general scheme. However, this position would be challenged increasingly by a minority trend which emerged in the nineteenth century, even though the evidence shows that it had existed earlier in a latent form. Consisting principally of laymen, it held that the death of an animal is usually unjust and this should be compensated for by some form of afterlife.

Citer ce document / Cite this document :

Baratay Éric. La mort de l'animal dans l'imaginaire catholique (France, XVIle-XXe siècle). In: Revue de l'histoire des religions, tome $212 n^{\circ} 4,1995$. pp. 453-476.

doi : 10.3406/rhr.1995.1251

http://www.persee.fr/web/revues/home/prescript/article/rhr_0035-1423_1995_num_212_4_1251 
ÉRIC BARATAY

Université Jean-Moulin Lyon 3

\section{LA MORT DE L'ANIMAL DANS L'IMAGINAIRE CATHOLIQUE (France, $\mathrm{XVII}^{\mathrm{e}}-\mathrm{XX}^{\mathrm{e}}$ siècle)}

Pour la majorité des catholiques, et notamment du clergé, la mort de l'animal n'est qu'un phénomène banal. Son absence évidente de survie permet de le tuer sans appréhension pour s'en servir abondamment. Les recommandations sporadiques sur la modération de la violence ne modifient pas ce schéma général.

Ce modèle est de plus en plus contesté par un courant minoritaire qui émerge au XIX siècle, même si des témoignages montrent qu'il existait auparavant à l'état latent. Composé essentiellement de laïcs, il considère que la mort de l'animal est le plus souvent injuste et qu'une forme de survie doit la compenser.

\section{The death of an animal in Catholic Imagination (France, 17th - 20th centuries)}

For most Catholics, in particular the clergy, the death of an animal is no more than a commonplace event. Since it obviously has no life after death it can be killed without apprehension and used in abundance. The occasional recommendation to use less violent methods of killing did not alter this general scheme.

However, this position would be challenged increasingly by a minority trend which emerged in the nineteenth century, even though the evidence shows that it had existed earlier in a latent form. Consisting principally of laymen, it held that the death of an animal is usually unjust and this should be compensated for by some form of afterlife. 


\section{Une mort normale}

A la différence de celle de l'homme, la mort de l'animal n'a jamais provoqué un grand intérêt. Elle apparaît comme un phénomène normal, comme une conséquence obligée, et sans rémission, de sa nature supposée. Car, si l'homme est considéré comme un composé de matériel et de spirituel, où celui-ci doit l'emporter grâce au passage par la mort salvatrice, la bête est une créature entièrement corporelle pour laquelle le dualisme chrétien esprit-matière n'existe pas.

Cette certitude ne vacille jamais et s'insère au cœur des philosophies qui se succèdent tout au long de l'histoire du catholicisme ${ }^{1}$. Du XIII ${ }^{\mathrm{e}}$ siècle jusqu'à la fin du $\mathrm{XVII}^{\mathrm{e}}$ siècle, la plupart des clercs souscrivent à l'idée thomiste d'une âme sensitive, cause interne de la vie et du mouvement. Une substance définie avec peine, mais dont la nature matérielle n'est jamais mise en doute. L'évidence est encore plus forte avec la théorie cartésienne de l'animal-machine. Entrevue par certains clercs de la première moitié du XVII ${ }^{e}$ siècle (le jésuite Étienne Binet en 1625, le minime Marin Mersenne en 1636) ${ }^{2}$, formalisée par Descartes dans le Discours de la méthode (1637), elle suscite un long débat dans le dernier tiers du siècle, notamment dans l'Église où s'affrontent ses partisans les plus zélés (Antoine Arnauld, Nicolas Malebranche) et ses adversaires les plus résolus (les jésuites). Bien que d'illustres personnalités n'osent pas prendre partie (Bossuet,

1. Le monde clérical constitue l'élément central de l'étude. La position des laïcs (signalés dans les notes par le signe *) est évoquée lorsqu'elle diverge du modèle dominant, ce qui est le cas, pour une minorité, à partir du xix siècle. La période choisie permet un panorama des mentalités allant des sensibilités médiévales, encore présentes au XVII siècle, aux conceptions les plus contemporaines. Pour une étude détaillée, voir Éric Baratay, L'Église et l'animal du XVIr' siècle à nos jours en France, thèse, Lyon III, 1991, à paraître au Cerf en 1996.

2. Essais des merveilles de nature, Rouen, éd. 1625, p. 76; Harmonie universelle, Paris, 1636, p. 79-80. 
Fénelon $)^{3}$, elle se diffuse peu à peu et se voit adoptée par la grande majorité du clergé dans les années 1670-1820 afin de répondre aux libertins du $\mathrm{XVII}^{\mathrm{e}}$ siècle, puis aux philosophes des Lumières qui remettent en cause le statut de l'homme en le rapprochant de la bête. Car, en réduisant l'univers à deux constituants irréductibles (la matière et l'esprit), cette théorie rejette toute possibilité d'une âme sensitive en l'animal et transforme celui-ci en une simple mécanique dotée de rouages (le cœur, le cerveau..), de ressorts (les muscles) et de fluides (le sang), évoluant comme un automate ${ }^{4}$. Même les thèses plus favorables aux bêtes s'inscrivent dans ce schéma. Pour mieux lutter contre un matérialisme toujours présent au début du $\mathrm{XIX}^{\mathrm{e}}$ siècle et pour sauver le statut privilégié de l'homme en démontrant qu'il possède bien une âme spirituelle, les clercs des années 1820-1870 prennent le contre-pied du cartésianisme et accordent aussi une âme spirituelle à l'animal, mais d'une nature très inférieure, bornée aux sensations, incapable de raison et surtout liée à la matière ${ }^{5}$. Le caractère peu orthodoxe de cette opinion explique le retour rapide au thomisme à partir des années 1870 , une conception encore en place de nos jours.

Si la mortalité définitive des bêtes est justifiée par les philosophies, elle constitue en réalité leur soubassement idéologique, car elles doivent absolument respecter cette condition, ce qui oblige à des contorsions intellectuelles (telle cette âme spirituelle et mortelle de la première moitié du XIX siècle) ou à des changements de cap lorsqu'elles n'apparaissent pas

3. Antoine Arnauld, Pierre Nicole, La logique, Paris, éd. 1965, p. 226 ; Nicolas Malebranche, De la recherche de la vérité, Paris, éd. 1963, p. 394 ; Jacques Bossuet, De la connaissance de Dieu et de soi-même, Paris, éd. 1873, p. 170-207; François Fénelon, Traité de l'existence et des attributs de Dieu, dans CEuvres, éd. 1820 , t. 1 , p. 36 et $\mathrm{s}$.

4. Voir l'exemple d'Antoine Dilly, De l'âme des bêtes, Lyon, 1676, p. 28-71, 206-227, 278. Sur la théorie cartésienne, voir Annie Bitbol-Hespéries, Le principe de vie chez Descartes, Paris, Vrin, 1990; Francisque Bouillier, Histoire de la philosophie cartésienne, Paris, 1868; Geneviève Rodis-Lewis, L'Anthropologie cartésienne, Paris, PUF, 1990. Sur son écho dans l'Église, voir Éric Baratay, op. cit., chap. 6.

5. Pierre Debreynne, Pensées d'un croyant catholique, Paris, éd. 1840, p. 187, $191,211$. 
solides. Cette conviction n'est pas fondée sur la Bible, qui n'a pas de position tranchée en ce domaine (cf. Qo, 3-19 et 21), mais sur la crainte d'une égalisation des créatures, d'une remise en cause du statut de l'animal et de sa place dans la religion. En 1876, l'abbé Beney, aumônier de l'hospice d'Autun, s'emporte contre ceux qui accordent une âme spirituelle aux bêtes: "Voilà les animaux irraisonnables élevés à notre niveau, ou, ce qui ne serait pas moins épouvantable, nous voici nous-mêmes abaissés, avilis, dégradés. (...) Si donc vous voulez que les âmes des bêtes survivent à la dissolution du corps (...) c'est-à-dire qu'elles soient immatérielles, intelligentes et libres, il faut encore que, même ici-bas, vous regardiez comme immatérielle, intelligente et libre l'âme du cheval qui vous sert, du chien qui vous caresse, de l'insecte que vous repoussez loin de vous (...). La conséquence est monstrueuse, direz-vous! C'est vrai; mais elle est nécessaire. (...) Accorder à une âme d'être immortelle (...) c'est la regarder comme une image de Dieu, c'est la croire capable de l'atteindre un jour par la connaissance et l'amour parfaits, c'est enfin lui promettre ce que Notre-Seigneur n'a promis qu'aux hommes, lorsqu'il leur adressait ces paroles: Vous serez semblables aux anges. $"{ }^{6}$ L'affirmation de l'entière mortalité des bêtes est l'expression d'une défense du statut de l'homme; elle constitue l'un des soubassements idéologiques de sa domination sur le monde et l'une des contributions de l'Église à la mentalité anthropocentrique de la société occidentale.

Car la mort de l'animal doit servir l'homme, considéré comme le microcosme récapitulant toutes les créatures, le centre du monde unissant le ciel et la terre en sa personne (la seule capable d'établir un lien avec Dieu en faisant office de prêtre de la création), le roi du monde terrestre, un titre que Dieu semble lui accorder lors de deux épisodes de la Genèse $(1-26,28 ; 2-20)$ toujours lus d'une manière littérale. Il s'agit du commandement divin de dominer les animaux, puis de

6. Lettre critique. L'immortalité de l'âme des bêtes jugée par saint Thomas d'Aquin, Autun, 1876, p. 78 et s. 
leur nomination par Adam. Les connaissant immédiatement, il en est bien le maître. La conviction que l'homme est la finalité de l'animal est particulièrement forte au XVIII ${ }^{\mathrm{e}}$ siècle. Fénelon affirme en 1712 que le chien est né pour le caresser, pour lui garder ses biens et pour attraper d'autres bêtes. Le cheval a été créé pour le porter et le soulager dans sa faiblesse. Quant aux moutons, ils «ont dans leur toison un superflu qui n'est pas pour eux, et qui se renouvelle pour (l')inviter (...) à les tondre toutes les années ». Ce sentiment permet d'exiger beaucoup des bêtes. Le bénédictin Calmet écrit en 1713 que les hommes ont «un pouvoir absolu (...) sur leur corps, sur leur vie » et l'abbé Moigno, chanoine de SaintDenis, note en 1877 qu'ils ont des «droits " ${ }^{7}$. Au XX siècle, la révélation de l'immensité des temps géologiques, de l'antériorité de la flore et de la faune par rapport à l'homme discrédite ce finalisme primaire. Mais il reste que l'homme doit dominer la nature. Cette exigence est renforcée par l'adoption d'une conception évolutionniste du monde à partir des années 19301940: dans sa marche vers Dieu, l'homme doit maîtriser une création en évolution et l'orienter en fonction de ses propres buts, devenant ainsi un cocréateur avec Dieu. La domination est érigée au rang d'un programme inscrit dans la marche du monde, donc normal et irréversible.

Tout cela justifie le droit de tuer. La chasse est longtemps perçue comme le moyen de retrouver la domination perdue à la suite du Péché originel, les animaux s'étant rebellés contre l'homme déchu. Elle comporte aussi des aspects pratiques (trouver des peaux et des vivres, apprendre des ruses de guerre, réveiller les corps engourdis) et constitue l'une des rares distractions qui ne mène pas au péché. Le jésuite Étienne Binet la qualifie, en 1621 , de plaisir «innocent» et «pur», affirmant, à propos des chasseurs, que «tout leur péché consiste à tuer un lièvre et désespérer un pauvre cerf $»^{8}$.

7. Traité de l'existence et des attributs de Dieu, Paris, éd. 1820, p. 31-32; Commentaire littéral sur les psaumes, Paris, 1713, t. 1, p. 77-78; Les splendeurs de la foi, Paris, 1877-1882, t. 2, p. 482.

8. Essais des merveilles de nature, Rouen, éd. 1625, p. 1-4. 
$\mathrm{Au} \mathrm{XVII}^{\mathrm{e}}$ siècle, elle semble tellement normale que des clercs la pratiquent assidûment. De 1636 à 1655, Armand de Rancé, futur réformateur de la Trappe, est un fervent chasseur: entre «les plaisirs qui l'emportaient alors, la chasse levait le premier rang; il y passait les jours et les nuits, couchant souvent dans les bois nue tête, accablé de fatigues. Il en remporta même une goutte sciatique, qui lui pensa faire perde la vie par l'excès de la douleur». De son côté, l'évêque de Saint-Flour, Mgr de Montrouge, est plus expert en vénerie qu'en théologie'. Cette participation ne régresse qu'à partir de la seconde moitié du $\mathrm{XVII}^{e}$ siècle, pour quasiment disparaître au XVIII ${ }^{\mathrm{e}}$ siècle sous l'action d'une hiérarchie acquise à la réforme catholique. La considérant désormais comme une activité profane détournant le bon prêtre de ses occupations spirituelles, elle l'interdit vigoureusement.

Bien que l'ordre de Dieu (Gn, 9-3) n'intervienne qu'après le Déluge, beaucoup conçoivent la consommation des viandes comme la conséquence du Péché originel qui a corrompu toutes les créatures. Le soleil ayant perdu de sa force, les fruits, trop altérés, furent complétés par une nourriture plus forte ${ }^{10}$. Cette interprétation est majoritaire jusqu'au $\mathrm{XX}^{\mathrm{e}}$ siècle, mais elle suscite quelques doutes. Pour certains, l'alimentation carnée est si naturelle qu'elle ne peut remonter qu'à la création de l'homme. L'oratorien Jacques Joseph Duguet écrit en 1732 que Dieu n'a jamais interdit cet usage : l'ordre de dominer les poissons et les oiseaux le sous-entend, car à quoi serviraient les bêtes «qui sont inutiles à l'homme, s'il n'a aucun droit sur leur vie " ?" A ce finalisme anthropocentrique s'ajoute l'idée du caractère naturel de la pratique, les animaux s'entre-dévorant. Cette conception s'impose au $\mathrm{XX}^{\mathrm{e}}$ siècle sous l'influence d'une science évoquant l'antériorité des vivants par rapport à

9. Pierre Le nain, La vie de Don Armand Jean Le Bouthiller de Rancé, s.1., 1715, t. 1, p. 14-15; Pierre Pierrard, Le prêtre français, Paris, Bloud et Gay, 1969, p. 40. p. 49.

10. François Placet, La corruption du grand et du petit monde, Paris, éd. 1668, 195.

11. Jacques Duguet, Explication du livre de la Genèse, Paris, 1732, t. 1, p. $192-$ 
l'homme et la lutte générale pour l'existence. La consommation de viande devient une conséquence normale de la loi de la vie.

C'est pourquoi les clercs considèrent sans cesse, en s'appuyant sur la Bible (Lc, 10-7; $1 \mathrm{Tm}, 4-1$ ), que le refus des viandes est condamnable. Le végétarisme est une superstition digne des peuples sans religion ou croyant à la détestable métempsycose. Cette situation perdure au $\mathrm{XX}^{\mathrm{e}}$ siècle, même si les convictions se font moins fortes, même si certains missionnaires aux Indes adoptent le végétarisme pour mieux se fondre dans les populations.

Ces exemples montrent que la mort de l'animal ne pose pas de problème de conscience. L'abbé Martinet résume le sentiment général lorsqu'il affirme, en 1850, que le fossé avec l'homme est tel que le sort de l'animal n'a guère d'importance : «Dieu met (...) la même distance que vous mettez entre le meuble sorti de vos mains et votre enfant. Vous sacrifieriez des milliers de meubles, fruit de votre industrie, pour sauver le fruit de votre amour, cet autre vous-même. $\gg^{12}$

Mais cela suppose la négation ou la justification de la souffrance des bêtes. L'un des postulats de la théorie de l'animalmachine réside dans le refus de la douleur chez les animaux. Un animal crie, non pas parce qu'il a mal, mais parce que sa machine produit des mouvements contraires. D'ailleurs, les animaux n'ayant pas chuté, il est impossible qu'un Dieu juste, bon et tout-puissant ait produit une créature sujette à la douleur, qui est l'état de punition des péchés. Toute compassion sous-entendrait qu'il est injuste et que les pratiques humaines sont scandaleuses, ce qui serait remettre en question la religion et la place de l'homme dans la création ${ }^{13}$.

Si les clercs des années 1820-1870 reviennent aux conceptions antérieures au cartésianisme (bien qu'imposée à l'animal à la suite du Péché originel, la souffrance fait partie de l'ordre terrestre), une autre explication s'impose dans les décen-

12. La science de la vie, Paris, 1850, t. 1, p. 264.

13. Nicolas Malebranche, De la recherche de la vérité, Paris, éd. 1963, t. 2 , p. 391. 
nies 1880-1930 sous l'influence des découvertes paléontologiques montrant l'antériorité des espèces carnassières par rapport à l'homme et à la chute. Celle-ci a défait la paix générale imposée par Dieu et libéré la férocité innée des animaux. La souffrance devient un aspect normal de leur nature vivante et organisée. Avec la vie, la mort fait nécessairement son apparition. Dans ce contexte, la plupart des clercs minimisent la souffrance. Parce qu'elles n'ont pas comme l'homme, écrit le théologien Camille Sadet en 1927, « un tissu organique d'une infinie délicatesse et un tissu nerveux d'une admirable unité ", parce qu'elles ne prévoient pas et ne se rappellent pas ce qui leur arrive, les bêtes souffrent moins que lui. Il se produit même un équilibre des peines et des bonheurs: "Une joie courte mais d'une intensité très vive, peut compenser les douleurs longues et lancinantes. " A l'homme, la vraie souffrance et la compensation dans l'au-delà ; aux bêtes, une vie neutre qui s'achève logiquement avec la mort: "L'homme a horreur de la mort violente, parce qu'elle lui semble anormale, qu'elle s'accompagne de tout un cortège de douleurs non prévues, et qu'elle répugne par trop à la délicatesse de son cœur et de ses sentiments. L'homme a horreur de la mort même naturelle, parce qu'il a l'amour de la vie, la soif de l'éternité (...). Il n'en va pas ainsi de l'animal qui n'est point du tout troublé par la perspective de la mort, pour l'excellente raison que cette éventualité ne s'impose jamais à lui. Qu'il succombe prématurément (...) qu'importe! c'est la fin d'une existence dont il n'avait nullement apprécié toute la valeur, puisque, à aucun moment, il n'en avait pris totalement conscience. ${ }^{14}$

A partir des années 1940, cette conception est doublée d'une argumentation basée sur la notion d'évolution. L'histoire de la création étant conçue comme une marche dirigée vers l'apparition de l'homme, puis vers la rencontre de celui-ci avec Dieu, la souffrance animale s'intègre dans ce mouvement général de transformation et s'avère nécessaire: «On n'arrête pas

14. Un angoissant problème. Si Dieu existe, pourquoi le mal?, Avignon, éd. 1927, p. 79, 33, 89, 94. 
une armée en marche vers la victoire pour éviter des plaies aux combattants ", écrit le dominicain Sertillanges à la fin des années $1940^{15}$.

Ainsi, la chasse n'est plus perçue comme un moyen de restaurer l'empire de l'homme sur les bêtes, car l'image du Péché originel s'efface, mais comme un facteur de la nécessaire maîtrise d'un monde en évolution. Son statut connaît d'ailleurs une revalorisation significative, puisque les interdictions concernant les clercs disparaissent. En 1921, une décision de la Sacré Congrégation du concile supprime toute peine de suspense pour fait de chasse, hormis celle à courre ${ }^{16}$. Les prélats entérinent le fait à partir de la décennie 1950 : les statuts synodaux n'évoquent plus la question et laissent toute liberté aux clercs. La situation est tacitement confirmée par le Code de droit canonique dans sa nouvelle version de 1983 (canon 285). Aussi les campagnes assistent-elles au retour des curés chasseurs. Outre le fait qu'ils considèrent la chasse comme un moyen de rapprocher l'Église de la société, d'investir celle-ci pour la rechristianiser, elle leur semble une réponse à l'ordre de Dieu de dominer les animaux. Les tuer ne pose pas de problème. Jugée compatible avec leur état, la chasse est un sport, une détente, un loisir comparable à la pelote basque, aux jeux de boules ou de cartes ${ }^{17}$.

Même évolution à propos de la corrida. Alors que les évêques du $\mathrm{XIX}^{e}$ siècle avaient violemment combattu son introduction, comme nous le verrons, les positions évoluent: les condamnations perdurent jusqu'aux années 1960, mais les arguments changent peu à peu. La dépravation de l'homme et la cruauté envers l'animal ne sont plus évoqués à partir de la décennie 1950. A l'inverse, l'idée d'une maîtrise de la nature se répand, certains mentionnant, voire reconnaissant l'art et l'adresse des toreros, image de l'intelligence de l'homme face à la force brute. Les interdictions d'assister se transforment alors

15. Antonin Sertillanges, Le problème du mal, Paris, éd. 1951, t. 2, p. 125-127. 311.

16. Raoul Naz (dir.), Traité de droit canonique, Paris, éd. 1954, t. 1, p. 310-

17. Entretiens dans Plaisirs de la chasse, Paris, novembre 1986, p. 23-36. 
en recommandations évasives ${ }^{18}$. A partir du concile Vatican II, l'article du Code de 1917 concernant la corrida tombe en désuétude. Une liberté d'attitude s'installe, entérinée par le nouveau Code de 1983. Dès lors, des clercs assistent aux corridas, tel l'évêque de Nîmes qui déclare en 1981: «Ce que je trouve très beau dans la corrida, c'est que l'homme cherche à maîtriser la bête, à maîtriser la nature. Cela a toujours été la vocation de l'homme tel que Dieu le veut (...). Tout ce qui permet à l'homme de maîtriser les choses, de maîtriser ce qui n'est pas lui, ce qui n'est pas humain, tout cela me semble aller dans le sens de la grandeur de l'homme. ${ }^{19}$

\section{Une mort quelquefois cruelle}

Certaines réserves sont cependant émises sur le droit de tuer. Au XVII ${ }^{\mathrm{e}}$ siècle, quelques clercs soulignent que le meurtre gratuit est condamnable, non pas pour l'animal lui-même dont le sort importe peu, mais pour le fait que le sujet concerné risque de devenir "un homme de sang et un meurtrier d'homme ". Ce souci se retrouve à propos des pratiques légitimes. Le curé Gruau, théoricien de la chasse, reconnaît en 1613 que "par l'effusion continuelle du sang des bêtes, les hommes sont faciles et enclins à commettre meurtres et cruautés ». Le regarder couler, c'est s'apprêter à contempler celui de l'homme. La même réserve est quelquefois émise au nom du respect de l'œuvre de Dieu que l'homme ne peut détruire sans vergogne $^{20}$. Cette modération est présente jusqu'au $\mathrm{XIX}^{\mathrm{e}}$ siècle, mais avec une intensité variable: toujours sporadique, elle est très rare à l'époque du cartésianisme.

Une argumentation centrée sur l'animal s'ajoute à celles-là dans les années 1850-1930, notamment à l'occasion de l'intro-

18. Catholicisme, hier, aujourd'hui, demain, Paris, 1952, t. 3, col. 219.

19. Corrida, novembre 1981 , p. $28-30$.

20. Julien Hayneufve, Le grand chemin qui perd le monde, Paris, 1646, p. 46 (citation); Louis Gruau, Nouvelle invention de chasse, Paris, éd. 1888, p. 16; Germain Habert, La vie du cardinal de Bérulle, Paris, 1646, p. 706. 
duction de la corrida espagnole. Adoptant la position de Pie V, dont la bulle De salute gregis (1567) vilipendait les courses de taureaux et interdisait toute présence de clercs, l'épiscopat proteste avec vigueur. A l'occasion de la première corrida avec picadors et mise à mort de taureaux à Nîmes en 1853, l'évêque du lieu se scandalise de cet attrait pour le sang, de ce dévoiement des chrétiens. Il proclame sa crainte d'un retour au paganisme et affirme qu'un chrétien "doit s'interdire non seulement de torturer, mais même de froisser, mais même d'inquiéter un être quelconque pour se faire un divertissement de ses tristesses $"{ }^{21}$. Les condamnations suivantes, celles du nouvel évêque de Nîmes en 1885 ou des prélats des années 1930 confrontés aux tentatives d'implantation de la tauromachie dans la France du Nord, rassemblent elles aussi le souci d'éviter une dégradation morale de l'homme, dangereuse pour luimême, et la compassion pour l'animal. Dans les années 1930, c'est même ce dernier motif qui prévaut : «Que l'on éprouve du plaisir, écrit l'évêque de Limoges en 1935, à ces corridas sanglantes qui consistent à traquer sur une arène de pauvres bêtes qui n'en peuvent plus, à les blesser cruellement, à les mettre à mort, mais pas trop tôt et pas trop vite, afin que le spectateur ait le temps de jouir, parfois à les massacrer inhumainement, voilà ce que nous n'arrivons pas à comprendre. $\|^{22}$

C'est justement à cette époque que le souci de modération s'officialise en quelque sorte, en partie sous l'influence d'un second courant que nous verrons plus loin, mais tout en restant prudent (le cruel est défini à l'aune de l'utilité anthropocentrique) et minoritaire. Des traités de théologie demandent que l'on évite la cruauté et les "souffrances inutiles». Les Scouts de France, fondés en 1920, se proposent d'apprendre le respect des créatures de Dieu. Le premier catéchisme national de 1937 comprend une question sur la souffrance animale : « Est-il permis de faire souffrir sans raison les animaux? Non, il n'est pas

21. Claude Plantier, Instructions, lettres pastorales et mandements, Nîmes, 1867, t. 1, p. 231-249. Voir Éric Baratay, Élisabeth Hardouin-Fugier, La corrida, Paris, PUF, 1995.

22. Semaine religieuse de Limoges, 16 juin 1935. 
permis de faire souffrir sans raison les animaux : ce serait un acte de cruauté. ${ }^{23}$

Mais, parce qu'ils veulent s'engager entièrement dans les problèmes sociaux et investir la société pour la rechristianiser, parce qu'ils mettent l'accent sur la priorité de la relation entre les hommes et sur la maîtrise de la nature, les clercs se désintéressent de ces premières réflexions après la seconde guerre mondiale. La leçon du catéchisme de 1937 disparaît de la version de 1967. Les traités de morale et les Scouts de France abandonnent ces thèmes dans les années 1950-1970. Le monde catholique revient à la conception d'une mort à volonté. Il faut attendre la décennie 1980 pour que des réserves réapparaissent, à une époque où l'Église cherche à reprendre en compte les aspects délaissés dans les dernières décennies. Des prélats s'élèvent de nouveau contre la chasse à courre ou la corrida. Les cardinaux Decourtray et Lustiger soutiennent en 1988 que l'homme ne doit pas tyranniser les animaux et que le respect de la vie ne se divise pas ${ }^{24}$. Bien que d'un avenir incertain, ces quelques positions reflètent un lent glissement d'opinion à l'intérieur du modèle majoritaire et, surtout, l'influence progressive d'une autre mentalité pour qui la mort de l'animal est souvent injuste.

\section{Une mort injuste}

Ce sentiment reste longtemps confiné dans le domaine des convictions intimes. Lorsqu'en 1621, l'abbé Michel de Marolles doit participer à une chasse à courre sur les terres paternelles, il use d'un subterfuge pour ne pas prendre part au groupe et n'ose qu'une discrète confidence sur son aversion pour ce genre d'exercice ${ }^{25}$. Il faut attendre les débuts du $\mathrm{XIX}^{\mathfrak{e}}$ siècle, et l'inscription d'un sujet sur les «traitements bar-

23. Eugène Baudin, Cours de philosophie morale, Paris, 1936, p. 255-257 ; Jacques Sevin, Le scoutisme, Paris, éd. 1924, p. 40 ; Catéchisme à l'usage des diocèses de France, Marseille, éd. 1938, p. 109.

24. Église à Lyon, Lyon, 12 novembre 1988, p. 345 ; Entretien Tv à Entre chien et loup, 3 octobre $1988,18 \mathrm{~h} 30$.

25. Mémoires, Amsterdam, éd. 1740, t. 1, p. 92-94. 
bares exercés sur les animaux » au concours 1802 du prix de morale de l'Institut de France, pour que la question passe dans le domaine public et pour qu'un clerc, le modeste desservant Arbaud de la succursale de Villeneuve dans les Basses-Alpes, s'intéresse ouvertement à elle.

Effacée durant les premières décennies du XIX ${ }^{e}$ siècle, elle resurgie avec la création de la SPA en 1845 et le vote de la loi Grammont en 1850, la première loi de protection, non pas des animaux sauvages, car le droit de l'homme à prélever son dû sur la nature n'est pas encore remis en cause, mais des animaux domestiques soumis à la violence quotidienne ${ }^{26}$. Avec quelques laïcs, une petite fraction du clergé prend part à cette évolution dans les années 1830-1850: des prêtres de paroisses, tel le curé d'Ars, des théologiens, comme l'abbé Moigno, des prélats, tel le cardinal Donnet à Bordeaux. Ce courant très minoritaire connaît un essor relativement continu avec des mutations dans le discours, à la fin du XIX siècle, dans les années 1950 et la décennie 1970, qui l'éloignent du modèle dominant. Elles s'accompagnent d'un changement sociologique qui représente à la fois leur cause et leur conséquence : alors que les fondateurs du milieu du siècle sont le plus souvent des clercs, ce sont ensuite les laïcs qui sont les plus nombreux et qui avancent les idées nouvelles ${ }^{27}$.

Jusqu'à la fin du XIX siècle, ce ne sont pas les fondements de la relation hommes-animaux qui sont contestés, mais ses aspects concrets. L'homme a des droits étendus, il peut tuer et faire souffrir si nécessaire, mais il doit remplacer la cruauté, signe de la «barbarie » et « de la plus révoltante injustice ${ }^{28}$, par le respect et

26. Valentin Pelosse, Imaginaire social et protection de l'animal, Des amis des bêtes de l'an X au législateur de 1850, L'Homme, Paris, 1981, n. 4, p. 5-33, 1982; n. 1, p. 33-51; Éric Pierre, Une société sous la monarchie de Juillet : la SPA, Formation, idéologie, sociologie, Histoire et animal, Toulouse, IEP, 1989, vol. 1, p. 315-331; Maurice Agulhon, Le sang des bêtes. Le problème de la protection des animaux en France au XIX ${ }^{e}$ siècle, Romantisme, Paris, 1981, p. 81-109.

27. Une étude sociologique des auteurs ne donne pas d'indications précises. Toutes les tendances du catholicisme sont plus ou moins représentées, notamment dans le clergé de l'époque contemporaine. Des évolutions sont cependant sensibles; la prédominance des séculiers et la disparition des prélats entre les années 1920 et 1980 ; le poids croissant des femmes pour les laïcs.

28. Guillaume Chardon, Roi et non tyran, Paris, 1862, p. 22-27. 
la bonté. Par la suite, les fondements eux-mêmes sont remis en cause. Mgr Bolo, vicaire général de Laval, affirme en 1914 que l'homme n'est pas le maître omnipotent des animaux, mais un simple usufruitier à la domination limitée et modérée ${ }^{29}$. La volonté de rompre avec l'opinion courante s'impose dans les années 1970. Elle n'est plus considérée comme une vérité intangible, mais comme une conception philosophique historiquement datable, donc amendable et transformable. Le bon plaisir de l'homme et la mort de l'animal à discrétion sont refusés; l'idéologie de la maîtrise de la nature, en place depuis les années 1940, est fortement contestée, parce qu'elle valorise la ruse et la force ${ }^{30}$. Ces sentiments se fondent sur la certitude croissante, notamment depuis les années 1930-1970, d'une proximité de nature, d'une communauté des vivants qui nécessite le respect de la vie, la fraternité et la charité universelles, les devoirs de l'homme et les droits de l'animal.

Tout cela se traduit par le refus de pratiques normales pour la majorité des catholiques. La chasse n'est pas vraiment discutée aux $\mathrm{XVII}^{\mathrm{e}}$ et $\mathrm{XVIII}^{\mathrm{e}}$ siècles où les oppositions sont rares et dissimulées. Il faut attendre le concours de 1802 pour voir apparaître une réprobation. Dans le but de moraliser l'humanité, de la rendre moins cruelle pour elle-même, le desservant Arbaud préconise de la réduire aux seuls animaux malfaisants et de limiter le nombre des chasseurs ${ }^{31}$. Loin d'être totale, le saut de mentalité serait trop fort, la critique s'insinue par le biais d'un tri entre les animaux, d'une séparation entre nuisibles et non nuisibles, une distinction reprise tout au long du $\mathrm{XIX}^{\mathrm{e}}$ siècle. A partir des années 1930-1950, l'opposition est plus systématique. Sous l'influence de l'écologie, pour qui cette notion de nuisible n'a pas de sens, les protecteurs abandonnent les distinctions d'espèce et s'acheminent vers une condamnation globale: ne relevant plus de la nécessité dans les sociétés

29. Henry Bolo, Les animaux et le devoir chrétien, Paris, 1914, p. 4-10.

30. Jean Bastaire*, Une communion retrouvée, Droits de l'animal et pensée chrétienne, Paris, 1986, p. 49-52.

31. Arbaud, mémoire pour le concours de 1802, Archives de l'Académie des Inscriptions et Belles-Lettres, côte 1-H-8, manuscrit n. 17, p. 3-9. 
occidentales, la chasse n'est qu'un loisir basé sur le plaisir de tuer; elle doit disparaître ${ }^{32}$.

La corrida est condamnée dès son introduction, mais, à l'époque, les protecteurs ne se distinguent guère du reste des catholiques. Par contre, son changement de statut, à partir des années 1940, les marginalise. Et cela d'autant plus que leurs critiques (nombreuses dans les années 1970-1990 lorsque la corrida devient un phénomène médiatique alors que la zoophilie ${ }^{33}$ prend un nouvel essor) sont entièrement centrées sur le sort de l'animal : la corrida n'est ni le symbole de la maîtrise de l'homme, ni un art ; elle n'est que souffrance, sang et mort $^{34}$.

La vivisection constitue un tout autre problème. Non pas aux $\mathrm{XVII}^{\mathrm{e}}$-XVIII ${ }^{\mathrm{e}}$ siècles, où les témoignages sont rares parce qu'elle est encore limitée, mais à partir de François Magendie et de Claude Bernard qui l'érigent en instrument privilégié de la médecine expérimentale. Elle suscite alors de vives indignations : celles de Jules Michelet, de Victor Schoelcher, promoteur de l'abolition de l'esclavage des noirs et partisan de la loi Grammont, ou de Victor Hugo, président d'honneur de la Société française contre la vivisection fondée en 1882. Les catholiques zoophiles s'inscrivent dans cette opposition ${ }^{35}$. Mais à partir des années 1920, les progrès de la médecine, le triomphe de l'idéologie technicienne rendent cette attitude difficile. Alliant souffrance de l'animal et utilité pour l'homme, la vivisection déroute et semble obliger au compromis: elle est reconnue nécessaire, mais elle doit être contrôlée pour éviter les abus, notamment les expériences inutiles ou réitérées. Cette opinion médiane ne satisfait plus tout le monde à partir des années 1970 , une époque où le progrès à tout prix est remis en

32. Jean Bastaire*, art. cité, p. 52.

33. Nous employons les mots "zoophile», "zoophilie» non pas dans leurs sens actuels, péjoratifs, de quasi-bestialité, mais dans leurs sens premiers, datant du XIX ${ }^{\epsilon}$ siècle, époque de leur création, de défenseur et d'amour des animaux.

34. "Les propos du P. Roger Pestre sur les corridas», Nouvelles de Pech-Petit, juillet 1990 , p. 22.

35. Voir Jacqueline Lalouette, Vivisection et antivivisection au $x I x^{e}$ siècle, Ethnologie française, XX, 2, 1990, p. 156-165, et l'abbé Moigno, Bulletin de la SPA, Paris, 1868, p. 350. 
cause, où les problèmes d'éthique prennent une place importante dans la société, où la souffrance animale constitue le critère primordial de toute action protectrice. La volonté d'une limitation de l'expérimentation au profit des techniques de substitution se fait plus forte et s'accompagne de l'espoir d'un changement profond des mentalités ${ }^{36}$.

La consommation de viande pose progressivement un dilemme équivalent. Aux $\mathrm{XVII}^{\mathrm{e}}-\mathrm{XVIII}^{\mathrm{e}}$ siècles, les rares témoignages évoquent une certaine gène. Dans son mémoire confidentiel du début du XVIII ${ }^{\mathrm{e}}$ siècle, Jean Meslier, curé d'Etrépigny, confie son embarras à l'idée de tuer pour manger : « Je n'ai jamais rien fait avec tant de répugnance, que lorsqu'il me fallait dans certaines occasions couper, ou faire couper la gorge à quelques poulets ou pigeonneaux, ou qu'il me fallait faire tuer quelques porcs. Je proteste que je ne l'ai jamais fait qu'avec beaucoup de répugnance et avec une extrême aversion (...). Je hais de voir seulement les boucheries et les bouchers. " C'est à ce titre qu'il affirme son dégoût des multiples sacrifices évoqués par la Bible, en tire argument pour refuser le dieu cruel des chrétiens et avouer ses convictions matérialistes, ce qui illustre la marginalité de ces opinions ${ }^{37}$.

C'est pourquoi, ce malaise ne débouche pas immédiatement sur la volonté de ne plus tuer pour manger. Jusqu'au début du $\mathrm{XX}^{\mathrm{e}}$ siècle, les plus favorables aux bêtes considèrent que le végétarisme est une solution utopique, voire condamnable, puisque liée à des philosophies hérétiques prônant la métempsycose. Il s'agit plutôt de tuer avec précaution, une demande d'abord exprimée prudemment, sous le couvert de motifs plus larges. En 1802, Arbaud veut «inspirer du rebut pour le meurtre même des animaux » en montrant qu'il existe un lien avec les homicides: les sauvages, les romains, les assassins, les bouchers, les gens trop carnassiers ont des comportements cruels envers les hommes, alors que les peuples qui croient en la

36. Raymond et Madeleine Chasles*, De la bête à Dieu, s.1., 1949, p. 53 ; Jean Gaillard*, Les animaux, nos humbles frères, Paris, 1986, p. 112.

37. Mémoire, dans CEuvres complètes, Paris, éd. 1970, t. 1, p. 215-217. 
métempsycose et les végétariens sont doux envers autrui. C'est pourquoi, il demande, non pas d'interdire la viande, car «tout se révolterait contre cette disposition ", mais des mesures pour éduquer l'opinion, d'ailleurs adoptées quelques décennies plus tard : ne pas tuer et dépecer les animaux en public, ne pas exposer la viande dans les rues, tuer avec douceur et rapidité pour éviter de s'en amuser, interdire aux bouchers de paraître tachés

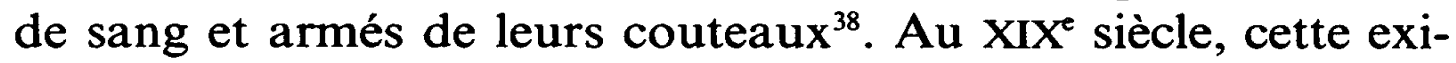
gence se centre peu à peu sur la souffrance de l'animal, puis elle s'étend, de nos jours, aux opérations précédant la mise à mort : l'élevage industriel, parce qu'il réduit les êtres vivants au rang de matière première, et le transport vers l'abattoir.

Ce n'est qu'au début du $X^{\mathrm{e}}$ siècle que le végétarisme apparaît comme une solution envisageable. Certains avancent que la consommation de viande est une conséquence du Péché originel que Dieu n'a pas voulu. S'en abstenir serait donc un moyen d'être plus proche de Lui. C'est, écrit Mgr Bolo en 1914, «ce que doivent vouloir le moins possible ceux qui tiennent à être fidèles à l'intention du Créateur ". Cette conception est pleinement soutenue dans la seconde moitié du $\mathrm{XX}^{\mathrm{e}}$ siècle où le végétarisme devient un idéal élevé vers lequel il faut tendre. Les textes montrent qu'il est pratiqué parmi les plus militants à partir des années $1970^{39}$.

En s'affirmant progressivement, cette sensibilité modifie ses justifications. Celles du XIX $X^{e}$ siècle étaient présentes auparavant, mais d'une manière sporadique. Elles ne se différencient du souci de modération quelquefois exprimé par le modèle dominant que par leur côté plus affirmé et systématique. En effet, elles oscillent entre un argument d'ordre religieux, le respect de la création de Dieu, et deux thèmes anthropocentriques: protéger l'homme lui-même en lui enlevant le goût du sang et de la cruauté; permettre que les bêtes mieux traitées rendent des services plus abondants.

38. Op. cit., p. 3-9, 15-19.

39. Henry Bolo, op. cit., p. 11, 121-123 ; Étienne Francois*, Bienfaits du végétarisme, Bêtes et Gens devant Dieu, Paris, octobre-décembre 1972, p. 5-6. 
Ces deux aspects sont progressivement abandonnés à partir des années 1890 et l'idée d'un respect de l'animal pour luimême s'impose dans la seconde moitié du $X X X^{e}$ siècle, particulièrement depuis 1970. Cette évolution est liée au fait que la souffrance des animaux devient le point central de la zoophilie. L'idée majoritaire d'une douleur minimale est de plus en plus considérée comme un paravent du bon plaisir de l'homme. Tous croient, au contraire, en une affliction proportionnelle au degré d'évolution de l'être vivant, ce qui veut dire que les animaux souffrent pleinement à leur manière. D'où le rejet des thèses cléricales sur l'origine de cet état (naturelle pour les bêtes, en résultat du Péché originel pour les hommes), car elles donnent à ces derniers une situation privilégiée que l'histoire dément et ne traduisent qu'un réflexe anthropocentrique. La souffrance animale n'était pas voulue par Dieu, mais a été introduite dès l'origine de la vie par Satan en prévision du Péché de l'homme. Bien que paradoxale et difficile à faire admettre dans une société qui n'évoque plus le démon, ni le Péché originel, cette conception est reprise par la plupart des protecteurs ${ }^{40}$. Elle permet de s'affirmer en accord avec la science tout en maintenant le rôle primordial de l'homme, afin qu'il ne se cache pas derrière la fatalité de la vie, change d'attitude et établisse de nouveaux rapports avec les bêtes.

Un autre thème s'impose en cette seconde moitié du $\mathrm{XX}^{\mathfrak{e}}$ siècle, dérivé de celui qui visait à rendre l'homme meilleur, mais transformé sous l'effet des mentalités communes. Parce que les deux créatures sont intégrées dans la même communauté des vivants, le respect de l'animal est considéré comme une facette du respect des autres. La manière dont sont traitées les bêtes correspond à la considération portée aux femmes ou aux autres peuples. Le spécisme semble de même nature que le racisme ${ }^{41}$.

40. Jean Gaillard*, op. cit., p. 32-33.

41. Aimé Michel*, Ce que je voudrais dire au Grand Corbeau, France catholique, Paris, 4 octobre 1985, p. 20. 


\section{Une mort attristante}

Ces diverses convictions s'accompagnent d'un sentiment d'affection pour les animaux. Jusqu'au XVIII ${ }^{\mathrm{e}}$ siècle, le seul admis, souvent réservé aux saints, est un amour général pour les créatures de Dieu. Mais de rares témoignages montrent qu'il existe un attachement plus profane, centré sur un animal de compagnie. Ainsi, Marie-Thérèse Brunet, religieuse hospitalière de l'Hôtel-Dieu de Chalon-sur-Saône vers 1773, se révèle inconsolable de la mort de son singe familier ${ }^{42}$. Cette sympathie apparaît véritablement dans les biographies, les souvenirs, les journaux personnels du XIX ${ }^{e}$ siècle. La dévote femme de Claude Bernard peuple son appartement de chiens et de chats. L'abbé Petit aime tout particulièrement son perroquet et lui dédie un poème lorsqu'il meurt en $1863^{43}$. Mais il existe, notamment chez les clercs, une gène face aux convenances sociales et les explications restent d'ordre religieux : par-delà l'animal, c'est le Créateur qui est aimét ${ }^{44}$. Cette réserve s'estompe à la fin du XIX $X^{\mathrm{e}}$ siècle pour les laïcs et au milieu du $X X^{e}$ siècle pour les clercs.

C'est pourquoi la perte de l'animal familier est de plus en plus vécue comme un drame et celui-ci est ouvertement exprimé en cette seconde moitié du $\mathrm{XX}^{\mathrm{e}}$ siècle. L'abbé Lévêque reconnaît en 1957 que «l'on éprouve semblablement auprès d'un animal ou d'un homme, mais pourvu qu'ils fussent aimés, le déchirement affreux qu'est la mort ». Cet état d'esprit n'est pas le fait des seuls militants. Les courriers des lecteurs de certaines revues catholiques montrent qu'il se développe en marge des mouvements officiels ${ }^{45}$.

42. Mémoire pour Jeanne Pusset (...) contre Sæur Marie-Thérèse Brunet, Dijon, s.d. (1773), p. 1-17.

43. Claude Bernard, Lettres beaujolaises, Villefranche, 1966, p. 141 ; Bulletin de la SPA, Paris, 1863, p. 84.

44. Jules Puiseux, Vie et lettres de Mgr Prilly, Châlons-sur-Marne, 1887, t. 1, p. 431-432.

45. Mon frère le chien, Paris, 1957, p. 22, 58; Le Gué, Paris, novembredécembre 1990, p. 28-29. 
Mais il pose d'une manière nouvelle, non plus seulement philosophique, la question de la destinée des bêtes. Les premières interrogations apparaissent dans la décennie 1830 et sont marquées par le tiraillement entre un sentiment personnel et la conception courante qui fait figure de dogme. "Je crois assez à l'âme des bêtes, écrit Eugénie de Guérin, et je voudrais même qu'il y eût un petit paradis (mais), il n'y a pas vouloir, c'est-à-dire choix, dans les actions animales, et, par conséquent, ni bien ni mal, ni paradis ni enfer. Je regrette cependant le paradis (...). Mon Dieu, qu'est-ce que je dis là ? Aurons-nous besoin de rien d'ici-bas, là-haut, pour être heureux?» Une ou deux décennies plus tard, les convictions sont plus fortes: en 1856, l'avocat Alexis Godin se dit certain de l'existence d'une vie future pour les bêtes ${ }^{46}$. Cette opinion fait vraiment irruption sur la scène publique à la fin du siècle, avec la Prière pour aller au Paradis avec les ânes de Francis Jammes, puis elle s'enracine profondément dans les mentalités protectrices de la seconde moitié du $\mathrm{XX}^{\mathrm{e}}$ siècle.

En soutenant l'immortalité de l'âme des bêtes, en dissociant l'affirmation de leur anéantissement du credo catholique, en affirmant que la Bible n'est pas formelle sur ce point, que l'Église n'a rien décidé officiellement et que le libre examen est possible, ces protecteurs remettent en question bien des certitudes. C'est pourquoi les clercs restent longtemps en retrait des laïcs. Ce n'est que dans les années 1890-1930 que certains prêtres osent poser le problème, puis que d'autres le dissocient du dogme pour le limiter à une simple affaire de sentiment ${ }^{47}$, une position habituelle depuis. La profondeur de la rupture avec le modèle commun explique aussi les hésitations sur la nature exacte de cette survie. Jusqu'aux années 1960, beaucoup avancent l'idée d'une simple résurrection lors du Jugement Dernier pour peupler la nouvelle terre promise par saint Pierre (2 P,

46. Journal, Paris, éd. 1874 , p. 85 ; Le protecteur, le législateur et l'ami des animaux, Paris, 1856, p. 287-288.

47. Paul Franche, La Légende dorée des bêtes, Paris, 1907, p. 14 ; Georges Fremont, Les principes, Paris, 1901-1906, t. 5, p. 104-108. 
3-13). Par la suite, la plupart des laïcs et nombre de clercs se rallient à la thèse d'une survie immédiate après la mort ${ }^{48}$.

Ces convictions sont fondées sur l'affection éprouvée envers les bêtes, qui conduit à refuser une séparation définitive, et sur la certitude du caractère injuste de leur souffrance. Victimes incessantes de l'homme depuis la Chute originelle sans que Dieu l'ait voulu, elles doivent revivre pour qu'elles aient droit à sa justice et que réparation leur soit donnée. Cette idée est fortifiée par l'image du Créateur qui s'impose à partir des années 1830-1840 sous l'impulsion de la piété ultramontaine. Au Grand Horloger, lointain et terrible du clergé jansénisant succède un «bon Dieu » plus proche, plus humain. D'autres justifications interviennent dans la seconde moitié du $\mathrm{XX}^{e}$ siècle, illustrant l'évolution des mentalités. Il s'agit du prix de la vie et du caractère immortel de l'âme des bêtes, des arguments provenant de la revalorisation de ces créatures et de la croyance d'une communauté avec l'homme ${ }^{49}$.

La survie n'a pas immédiatement concerné tous les animaux. Les interrogations naissent à propos du chien, le fidèle ami de la vie quotidienne. Les autres compagnons, tel le cheval, et les bêtes connotées positivement dans l'imaginaire catholique (les douces, les symboles du Christ ou de la Vierge) suivent de près. Eugénie de Guérin écrit qu'elle aimerait un paradis «pour les bonnes et les douces, comme les tourterelles, les chiens, les agneaux. Mais que faire des loups et autres méchantes espèces? Les damner? Cela m'embarrasse. L'enfer ne punit que l'injustice et quelle injustice commet le loup qui mange l'agneau? Il en a besoin (...). En suivant son instinct, elle (la bête) est bonne ou mauvaise par rapport à nous seulement ${ }^{50}$. L'éventail du bestiaire sauvé s'élargit ainsi progressivement. A la fin du XIX $X^{e}$ siècle, Francis Jammes évoque les animaux nuisibles et les maltraités: les veaux de boucherie, les

48. $1^{\text {er }}$ cas : Paul Chanson*, Les animaux sous l'arc-en-ciel, Paris, 1939, p. $122-135 ; 2^{e}$ cas : Jean Gaillard*, op. cit., p. $44,47$.

49. Alexis Godin*, op. cit., 1856, p. 15, 287-372; Michel Damien*, L'animal, l'homme et Dieu, Paris, 1978, p. 208.

50. Op. cit., p. 85. 
ânes des charrois, les lièvres abattus. Au XXe siècle, quelquesuns réservent encore ce privilège aux espèces les plus proches de l'homme, jugées les plus évoluées, mais la majorité proclame la survie de toutes ${ }^{51}$.

Face aux silences des Écritures, certains reconnaissent ne pas savoir quelle sera la nature de cet au-delà. Mais, pour la plupart, il s'agit d'un lieu particulier, conforme à leur état terrestre, permettant un dédommagement bien mérité des maux endurés sur terre et des services rendus. Dans Le Roman du Lièvre de Francis Jammes, chaque espèce a son propre paradis. Les chiens font ce qu'ils ont toujours aimé sur terre, les oiseaux chantent sans fin et sans peur, le paradis des brebis est un vallon d'émeraude, etc. Pour ces protecteurs, il n'est jamais question d'une quelconque vision de Dieu.

Mais d'autres l'envisagent. Précurseur très isolé en son temps, Alexis Godin croit qu'hommes et bêtes se retrouveront $^{52}$. Francis Jammes se rallie à cette conception dans sa Prière pour aller au Paradis avec les ânes. Elle se propage au $\mathrm{XX}^{\mathrm{e}}$ siècle, sans être majoritaire. Elle suppose que les bêtes soient près de Dieu, une idée qui ne déplaît pas au P. Pestre, curé d'une paroisse de Marseille: «La présence de mon chien contemplant, avec ses seules possibilités de connaissance, telles que Dieu les lui a données et appliquées à son maître sur la terre, troublerait-elle la mienne si je l'avais à côté de moi quand je serai moi-même auprès du Seigneur? ${ }^{53}$

Ces sentiments génèrent certaines pratiques. Eugénie de Guérin illustre leur apparition dans la décennie 1830: «Une de mes amies demandait une fois des prières pour son chien malade; je me moquai d'elle et trouvai sa dévotion mal placée. Aujourd'hui j'en ferais comme elle, je ne trouve pas cette prière si étrange tant le cœur change l'esprit! (...) ma conscience ne s'offusque pas d'intéresser le bon Dieu à la conservation d'une

51. Raymond et Madeleine Chasles*, op. cit., p. 114-118; Michel Damien*, op. cit., p. 202.

52. Op. cit., p. 736-737.

53. Les animaux ont-ils une âme?, Comité de défense des bêtes libres de SaintRoch, Marseille, février 1988, p. 5. 
bête. Y a-t-il rien d'indigne dans ses créatures, et ne peut-on pas lui demander la vie de celles que nous aimons? Je suis porté à le croire et qu'on peut, excepté le mal, tout demander à Dieu, au bon Dieu. ${ }^{54}$ En raison de ces incertitudes, la prière reste longtemps souterraine, hormis l'exemple célèbre de Francis Jammes. Elle fleurit ouvertement à partir des années 1970, obtenant une complète légitimité. Les clercs l'encouragent, les laïcs la pratiquent, l'Association catholique pour le respect de la création animale diffuse celles des adhérents. Ces textes retracent les convictions et les combats, s'adressent à Dieu et à François d'Assise pour convertir les hommes et maintenir les bêtes en bonne santéss.

L'idée de bénir les animaux familiers, en s'appuyant sur les traditionnelles bénédictions de troupeaux, apparaît au $\mathrm{XIX}^{\mathrm{e}}$ siècle. Eugénie de Guérin illustre encore cette naissance lorsque, assistant à l'une d'elle, elle regrette de n'avoir pas fait bénir son chien, mort quelques temps auparavant ${ }^{56}$. Mais cette cérémonie ne s'impose qu'à partir des années 1960-1970, à l'initiative de divers mouvements comme la SPA d'Alsace ou l'Association catholique pour le respect de la création animale. Comme les prières, elle mêle sentiments généraux (œuvrer pour la paix entre les vivants) et demandes concrètes de protection pour les animaux domestiques. La continuité revendiquée avec les anciennes bénédictions cache cependant un véritable changement : alors qu'il s'agissait de bêtes assimilées à des instruments de travail, comme l'on bénissait maisons, bateaux, récoltes, cela concerne maintenant des animaux reconnus êtres vivants à part entière et frères de l'homme. C'est à ce titre que resurgit une pratique disparue au XVIII ${ }^{e}$ siècle, celle des messes destinées à solliciter la bonté de Dieu. Le P. Pestre n'hésite pas, à la demande des fidèles, de célébrer pour la guérison d'animaux familiers.

De ces rites à l'enterrement des êtres chers, le glissement est insensible. La pratique émerge au $\mathrm{XIX}^{\mathfrak{e}}$ siècle, mais il n'est pas

54. Op. cit., p. 219, 234.

55. Bêtes et Gens.., Paris, $2^{c}$ trim. 1990, p. 6-9.

56. Op. cit., p. 219, 234. 
encore question de signes religieux. Eugénie de Guérin inhume son chien et l'une de ses tourterelles dans son parc, plantant des rosiers par-dessus. Montalembert fait de même pour son chien et Thérèse de Lisieux pour les oiseaux ${ }^{57}$. Au $\mathrm{XX}^{e}$ siècle, alors que l'hostilité de la majorité des catholiques reste sensible, les clercs protecteurs acceptent cet usage tout en maintenant une certaine orthodoxie: "L'Église, écrit en 1955 l'abbé Gautier, directeur du séminaire de Saint-Sulpice, n'autorise en aucune façon à placer sur le lieu où repose le corps d'un animal un emblème religieux. Par contre, on ne saurait blâmer ceux qui ornent de fleurs la tombe de leur chien, ce qui est une façon naturelle et touchante de marquer la pérennité du souvenir. ${ }^{58}$ Cette prudence ne peut endiguer l'évolution des mentalités. A Bessenay, dans le département du Rhône, 7 des 125 tombes d'un cimetière pour chiens, visité en 1986, sont décorées d'objets religieux: croix, personnages de la crèche, Vierge de Lourdes, anges. Au cimetière de Beaumont-sur-Lèze, en Haute-Garonne, le gérant raconte que les familles pleurent, se signent souvent au moment de l'inhumation et qu'un grand nombre revient fleurir les tombes à la Toussaint ${ }^{59}$. Des faits qui illustrent l'anthropomorphisation des animaux familiers et leur entrée dans l'économie du Salut.

74 rue Pasteur, 69007 Lyon

57. Eugénie de Guérin*, ibid., p. 220, 85; Juliette Goublet*, L'Église et le monde animal, Aurillac, éd. 1977, p. 94 (Montalembert); Thérèse de Lisieux, Histoire d'une âme, Paris, éd. 1979, p. 96 . On constate une évolution parallèle dans les milieux agnostiques: le premier cimetière pour animaux familiers est ouvert à Asnières en 1899 .

58. Un prêtre et son chien, Paris, 1955, p. $89,96$.

59. La voix des bêtes, Paris, janvier-février 1988, p. 26. 